\title{
Risposta al Padre R. Cantalamessa. Diffondere la conoscenza del dibattito esegetico su Gesù è oggi necessario
}

\section{Mauro Pesce}

Storicamente, 2 (2006).

ISSN: 1825-411X. Art. no. 62. DOI: 10.12977/stor544

II Padre Raniero Cantalamessa ha dedicato un lunghissimo articolo al libro Inchiesta su Gesù di Mondadori scritto da Corrado Augias (intervistatore) e da Mauro Pesce (intervistato). Lo scopo principale delle mie risposte ad Augias in questo libro è di esporre ad un pubblico vasto alcune delle questioni dibattute da decenni nell'esegesi di tutte le parti del mondo sulla base della competenza che mi sono fatto in quasi quaranta anni di studio. Leggo nell'articolo di Cantalamessa la forte preoccupazione che questa diffusione di opinioni esegetiche possa nuocere alla fede dei lettori. Da qui il bisogno che un ecclesiastico noto critichi questo libro in modo che i lettori siano vaccinati.

$\mathrm{Ma}$ io mi domando: quale tipo di fede è quella che vacilla di fronte all'esposizione di opinioni esegetiche? La ricerca storica - almeno la mia e di molti, molti esegeti oggi - non è né per la fede, né per la non-fede. Non nasce da una ragione corrosiva ed "incredula". Rivendico l'autonomia della ricerca dalle fedi e dalle non fedi.

II Padre Cantalamessa nell'intento di proteggere i lettori di «Avvenire» dal supposto pericolo rappresentato dal libro, mi attribuisce delle affermazioni che io non ho mai scritto, anzi, che ho esplicitamente criticato. Non credo che questo dipenda da una volontà di screditarmi ingiustamente, ma forse 
da una non perfetta conoscenza di una parte della ricerca esegetica e storica attuale, più che giustificata da parte di chi da tempo si occupa di altro.

Siccome l'articolo mi attribuisce - non solo una o due volte, ma in continuazione - dei pareri che io non ho mai sostenuto, sono costretto a ribadire quello che ho effettivamente scritto.

1. Cantalamessa scrive che io sarei «sulla scia» del // Codice da Vinci di Dan Brown. Mi stupisce l'affermazione del recensore. Ad una critica severa del libro di Dan Brown sono infatti dedicate le pagine 231-232 del libro. A mio parere, anzi, Cantalamessa non mette a fuoco il vero veleno di Dan Brown che è la falsificazione di tutti i documenti che fa finta di utilizzare e l'invenzione totale di un Gesù che considera un rito sessuale il centro dell'unione con Dio e concepisce tutta la storia in chiave occultista. La verità sarebbe per definizione occulta e solo una setta perseguitata la trasmetterebbe. Nessun esegeta professionista ha mai sostenuto questo e certamente non io e neppure Augias. La distinzione tra Gesù storico e Cristo della fede (che del resto mi appartiene poco) è cosa ben diversa dalle fantasie di Dan Brown.

2. Cantalamessa scrive: «II filone scelto è quello che va da Reimarus, a Voltaire, a Renan, a Brandon, a Hengel, e oggi a critici letterari e «professori di umanità», quali Harold Bloom e Elaine Pagels. Del tutto assente l'apporto della grande esegesi biblica, protestante e cattolica, sviluppatasi nel dopo guerra, in reazione alle tesi di Bultmann, molto più positiva circa possibilità di attingere, attraverso i Vangeli, il Gesù della storia». Questa affermazione deforma completamente quello che ho scritto. lo ho criticato Voltaire (pagine 47-48). II libro di Renan su Gesù non mi è mai piaciuto. Di Brandon ho scritto decine di pagine di critica aspra per dimostrare che la sua idea di un Gesù rivoluzionario politico-militare è esegeticamente infondata. Del libro di Bloom, non ho avuto il tempo che di sfogliare qualche pagina. Martin Hengel è uno studioso di fama mondiale che appartiene invece alla grande esegesi. Dire che la grande esegesi è «del tutto assente» dalle mie risposte è una 
vera e propria offesa. Sono stupefatto. Ho avuto come maestri Heinrich Schlier, Jacques Dupont e Rudolf Schnackenburg. Mi sono sempre ispirato a W.G. Kümmel e Ph. Vielhauer. Nei miei libri e articoli sul Vangelo di Giovanni e anche nel libro scritto con Augias tengo conto costantemente dell'esegesi che Raymond Brown e Schnackenburg hanno fatto del Vangelo di Giovanni, per non parlare di Theissen, Sanders, Dunn, Meeks, Milgron, Levine e infiniti altri. Ed è francamente assurdo rimproverare a me di ignorare Brown o Schnackenburg, visto che sono stato uno di quelli in Italia che li ha sempre utilizzati quando il defunto Padre de la Potterie al Pontificio Istituto biblico cercava di limitarne l'influsso, perché li riteneva troppo audaci, per non parlare del sospetto con cui investiva il Padre Boismard. Da più di vent'anni dirigo una rivista specialistica tra le più importanti di studi esegetici. Non riesco a capire perché il padre Cantalamessa voglia dare di me un'immagine così deformata.

3. Il padre Cantalamessa scrive: «All'uso selettivo degli studi corrisponde un uso altrettanto selettivo delle fonti. I racconti evangelici sono adattamenti posteriori quando smentiscono la propria tesi, sono storici quando si accordano con essa». Se questo fosse vero io sarei un esegeta poco serio e senza metodo. Al contrario, in quaranta anni di lavoro esegetico, ho elaborato una precisa e articolata criteriologia per la ricerca degli elementi più antichi e più vicini alla figura storica di Gesù. Era ovvio che nelle poche frasi di un'intervista non potevo ogni volta procedere alla dettagliata dimostrazione esegetica che esige decine e decine di pagine. II padre Cantalamessa ha tutto il diritto a non essere d'accordo con la mia esegesi: non lo chiedo neppure ai dottorandi in scienze bibliche da me diretti. Ma non può dire ai suoi lettori che infrango le regole elementari dell'onestà metodologica.

Ad esempio, io sostengo che la concezione del perdono dei peccati di Gesù è diversa da quella della chiesa primitiva. II testo che mi permette di attingere la visione di Gesù è una delle invocazioni del Padrenostro che ritengo assolutamente certo essere gesuano. La versione di Matteo delle 
parole dell'ultima cena mi sembra invece influenzata da una cristologia successiva, ma lo ritengo sulla base di un confronto dei racconti del battesimo da cui risulta che la frase «in remissione dei peccati» fu probabilmente tolta da Matteo al battesimo del Battista per attribuirla a Gesù. Ma è una ipotesi scientifica, fatta con metodo, e quindi verificabile (o falsificabile). 
4. Il padre Cantalamessa scrive che secondo me le «scoperte di nuovi testi ... avrebbero modificato il quadro storico sulle origini cristiane. Esse sono essenzialmente alcuni Vangeli apocrifi scoperti in Egitto a metà del secolo scorso, soprattutto i codici di Nag Hammadi». Anche questo non corrisponde a verità. Per me i testi gnostici di Nag Hammadi hanno ben poca importanza per il Gesù storico. II lettore vedrà che i cosiddetti apocrifi che a volte cito sono il Protoevangelo di Giacomo che sostiene la verginità di Maria, L'Ascensione di Isaia e la Didaché che non sono vangeli e non sono gnostici perché contengono tradizioni più antiche del Vangelo di Matteo, secondo la grande esegesi degli ultimi trent'anni. Cito poi il Vangelo di Pietro che, secondo storici molto cauti (e non solo secondo J.D. Crossan) contiene un racconto della passione che nella sua fase di redazione più antica potrebbe essere addirittura premarciano. Cito anche il Vangelo del Salvatore che non è gnostico da nessun punto di vista, ma non credo affatto che sia più antico del Vangelo di Giovanni. Lo cito semplicemente per informare il pubblico italiano di una scoperta molto importante della fine degli anni Novanta che non ha avuto alcuna ripercussione in Italia. Sul Vangelo di Tommaso la ricerca esegetica è molto più complessa di come la presenta il padre Cantalamessa e io mi sono limitato ad esporre un parere esegetico molto cauto, quello di J.D. Kaestli, che gode di fama inossidabile, il quale ritiene che alcune parti di Tommaso siano indipendenti dai Sinottici e altre no e che bisogna valutare caso per caso. D'altra parte i monaci cristiani antichi sembra che abbiano utilizzato per secoli il Vangelo di Tommaso come nutrimento spirituale, come emerge dagli scritti spirituali dello PseudoMacario.

Soprattutto io non credo affatto che il Gesù storico si trovi nei vangeli gnostici. Quando Augias mi ha interrogato sull'essenza del messaggio di Gesù ho risposto: «Luca è a mio parere colui che ha meglio compreso l'essenza del suo messaggio» (p.221).

Inoltre ho scritto: «altri danno credito a certi scritti apocrifi, negando quasi per principio ogni attendibilità ai testi canonici o alle affermazioni delle 
chiese» (p. 235). Perché dire al lettore che io ho scritto cose che non ho mai affermato e pensato?

II mio interesse per scritti non canonici del primo cristianesimo nasce negli anni Settanta quando volevo integrare la mia preparazione di specialista delle lettere di Paolo. Questo interesse si è incanalato nella Association pour l'Etude de la Littérature apocryphe chrétienne divenuta in trent'anni una delle organizzazioni scientifiche più serie in tutto il mondo. Questa associazione che raccoglie i migliori esegeti francesi, svizzeri, italiani, europei (e ora anche americani) ha letteralmente rinnovato lo studio del cristianesimo antico proprio grazie all'edizione critica e commento storico di un numero cospicuo di fonti trascurate come «apocrife», ma i testi gnostici sono stati del tutto marginali in questa associazione. Basti vedere le edizioni critiche e la raccolta in due grandi volumi presso la Pléiade.

5. Il padre Cantalamessa scrive: «i vangeli apocrifi professano tutti, chi più chi meno, una rottura violenta con l'Antico Testamento, facendo di Gesù il rivelatore di un Dio diverso e superiore». Mi dispiace doverlo contraddire, ma non è vero che «tutti» i vangeli apocrifi presentino una rottura violenta con le sacre scritture ebraiche. Molti vangeli apocrifi non sono gnostici e non presentano la contrapposizione tra messaggio cristiano e sacre scritture ebraiche che molti testi gnostici invece sostengono.

Ma poi, sia detto una volta per tutte, nel libro io utilizzo pochissimo gli scritti apocrifi. Li cito solo qua e là. Non capisco perché concentrare tanta attenzione su questo elemento marginale.

6. II padre Cantalamessa passa immediatamente dopo a parlare della «rivalutazione della figura di Giuda nel vangelo omonimo» e si domanda: «Si è disposti a seguire i vangeli apocrifi su questo loro terreno?». II lettore di «Avvenire» ha così l'impressione che io sostenga le teorie del Vangelo di Giuda. Nel libro invece io ho scritto: «Questo testo non ci offre alcuna notizia storica attendibile né sulla figura di Gesù né su quella di Giuda. È una specie di controvangelo, scritto per contestare i vangeli di Giovanni e di Matteo per condannare le idee e le pratiche religiose della Chiesa 
maggioritaria, che si rifaceva ai dodici apostoli» (p.229). In agosto 2006, ho pubblicato su una rivista francese un articolo in cui dimostro filologicamente che il Vangelo di Giuda dipende dai vangeli di Giovanni e di Matteo e forse anche da Marco e Luca, oltre che dagli Atti degli Apostoli e un mio articolo più argomentato è imminente presso la rivista «Humanitas». Come posso essere sospettato di attribuire attendibilità ad uno scritto del genere?

7. Il padre Cantalamessa mi accusa di non utilizzare Paolo per ricostruire la figura storica di Gesù. Scrive: «La sua testimonianza viene solo discussa a proposito della risurrezione, ma per essere naturalmente screditata». Mi domando: perché io screditerei «naturalmente» la testimonianza di Paolo? lo, come storico, nutrito di qualche conoscenza antropologica prendo molto sul serio quando un testo mi dice che si sono verificate delle apparizioni del risorto. Io credo che realmente Paolo e i primi discepoli ebbero delle apparizioni. E mi sono anche domandato in qual luogo, in quale zona di un grande edificio avesse potuto verificarsi un'apparizione a cinquecento persone. II padre Cantalamessa sembra irridere al tentativo di alcuni esegeti di interpretare le apparizioni come «stati alterati di coscienza». Questa espressione tecnica non significa affatto che una persona è «alterata». Significa solo che esistono stati di coscienza diversi dal normale. Questi esegeti, di cui io riporto solo l'opinione, ritengono che le neuroscienze permettano di capire come le apparizioni possano verificarsi e come entità «soprannaturali» possano entrare in contatto con la coscienza degli uomini e si rifanno anche alla teoria dei cosiddetti stati alterati di coscienza. Ma questa spiegazione può poi essere interpretata in due modi. Alcuni, come John Pilch della Georgetown University, che ne ha scritto più volte presso case editrici cattoliche americane, ritengono che le realtà soprannaturali entrino realmente in contatto con l'uomo. Altri ritengono che si tratti di un fenomeno psichico interamente autogenerato dal cervello umano e influenzato da logiche collettive. Io non ho preso posizione, perché non mi intendo di neuroscienze. Da storico che si interessa anche ad aspetti antropologici del cristianesimo primitivo mi è sembrato doveroso/importante 
informare i lettori di questo settore di ricerca, che offre materiale interessante al dibattito scientifico.

8. II padre Cantalamessa scrive: Secondo Pesce «il cristianesimo "nasce addirittura nella seconda metà del II secolo". Come conciliare quest'ultima affermazione con la notizia degli Atti degli apostoli $(11,26)$ secondo cui, non più di sette anni dopo la morte di Cristo, circa l'anno 37, "ad Antiochia per la prima volta i discepoli furono chiamati cristiani"»? Qui sono costretto a rimandare ai molti libri e decine di articoli che da venti anni discutono la questione. L'interpretazione che dà il padre Cantalamessa di questo testo è oggi variamente contestata. Rimando ai diversi contributi del volume di «Annali di Storia dell'Esegesi» dal titolo Come nasce il cristianesimo (Edizioni Dehoniane, Bologna, 2004), agli studi di Judith Lieu, ecc. Gran parte del problema sta nel precisare cosa si intende per cristianesimo da un punto di vista storico. L'apparire della parola cristianesimo del resto non è anteriore - allo stato attuale delle conoscenze - al primo decennio del II secolo.

9. L'idea che il cristianesimo fin dall'inizio presenti una pluralità di posizioni e che solo ad un certo punto si affermi un cristianesimo normativo è tesi storica che è ampiamente diffusa almeno dalla metà degli anni Trenta del XX secolo e che oggi mi appare largamente prevalente. Credo che proprio un patrologo cattolico, Alain Le Boulluec, abbia tempo fa mostrato come il concetto di eresia faccia la sua apparizione nel cristianesimo antico alla metà del secondo secolo e che il termine airesis assuma allora il significato negativo che ha poi assunto.

10. Un ultimo punto. II padre Cantalamessa mi accusa di sottolineare «sempre» le divergenze, e «mai» le convergenze tra i Vangeli canonici. In realtà, io sottolineo a volte differenze tra alcuni testi e a volte somiglianze tra altri testi. Somiglianze e dissimiglianze si aggregano e si disaggregano. lo non sono preoccupato di difendere l'unità del Nuovo Testamento, perché questa collezione di scritti è ben posteriore a Gesù, a Paolo e alla redazione 
dei primi vangeli, che è l'epoca che mi interessava nel libro.

II padre Cantalamessa dice concludendo che ci divide la fede. Non sono d'accordo. La fede non mi divide da nessuno. La ricerca storica non divide, se non da altre opinioni storiche. Ricondurre tutto a fede e non fede o addirittura fede-incredulità, significa compromettere un sereno e libero dibattito. La notizia dell'articolo polemico di Cantalamessa mi è giunta quando ero negli Stati Uniti per partecipare a quella che è forse la riunione principale di tutti i biblisti del mondo. In essa circa cinquemila docenti di Facoltà laiche o teologiche discutono con acribia, ma anche con estrema libertà, di ogni questione esegetica, senza nessuna censura o condanna. Questa appassionata ricerca, solida, documentata, arriva ormai anche al grande pubblico e continuerà sempre più ampia e inarrestabile anche nei prossimi decenni. Bisogna conoscerla, farla conoscere e - ovviamente scegliere criticamente ciò che ciascun competente ritiene valido. 